\title{
Collective synchronization in the presence of reactive coupling and shear diversity
}

\author{
Ernest Montbrió ${ }^{1}$ and Diego Pazó ${ }^{2}$ \\ ${ }^{1}$ Computational Neuroscience Group, Department of Information and Communication Technologies, \\ Universitat Pompeu Fabra, E-08003 Barcelona, Spain \\ ${ }^{2}$ Instituto de Física de Cantabria (IFCA), CSIC-Universidad de Cantabria, E-39005 Santander, Spain
}

(Received 3 August 2011; published 12 October 2011)

\begin{abstract}
We analyze the synchronization dynamics of a model obtained from the phase reduction of the mean-field complex Ginzburg-Landau equation with heterogeneity. We present exact results that uncover the role of dissipative and reactive couplings on the synchronization transition when shears and natural frequencies are independently distributed. As it occurs in the purely dissipative case, an excess of shear diversity prevents the onset of synchronization, but this does not hold true if coupling is purely reactive. In this case, the synchronization threshold turns out to depend on the mean of the shear distribution, but not on all the other distribution's moments.
\end{abstract}

DOI: 10.1103/PhysRevE.84.046206

PACS number(s): 05.45.Xt, 89.75.-k, 87.10.-e, 62.25.-g

\section{INTRODUCTION}

Reaction-diffusion systems consisting of a large number of degrees of freedom display a rich variety of dynamical regimes that are important in a wide range of fields [1-3]. In particular, systems composed of many interacting aggregates of heterogeneous, self-oscillating elements, often show oscillations at the macroscopic level as a consequence of the collective synchronization of the individual oscillators [1,4-6]. An appropriate model to study collective synchronization is the mean-field version of the complex Ginzburg-Landau equation (CGLE) with heterogeneity,

$$
\begin{aligned}
\dot{z}_{j}= & z_{j}\left[1+i\left(\omega_{j}+q_{j}\right)-\left(1+i q_{j}\right)\left|z_{j}\right|^{2}\right] \\
& +\frac{K}{N}(1+i c) \sum_{k=1}^{N}\left(z_{k}-z_{j}\right) .
\end{aligned}
$$

This equation describes an ensemble of $N \gg 1$ globally coupled limit-cycle oscillators, each defined by a complex variable $z_{j} \equiv \varrho_{j} e^{i \theta_{j}}$. Every oscillator differs from the rest in the natural frequency of rotation $\omega_{j}$ and in the shear (or nonisochronicity) $q_{j}$, which measures how the frequency of rotation depends on the oscillator's amplitude $\varrho_{j}$. Here we consider $\omega_{j}$ and $q_{j}$ to be independent random variables, with a joint probability function $p(\omega, q)=g(\omega) h(q)$.

The oscillators are coupled via a diffusive coupling of strength $K$, which has both a real (dissipative) and an imaginary (reactive) component. In general, a positive dissipative coupling drives the system to a more homogeneous state [7] (but see [8]). The effect of reactive coupling on synchronization is more intricate and strongly relies on the presence of shear $q_{j} \neq 0$ [9].

More than 30 years ago, the Kuramoto model (KM) was proposed as an analytically tractable system to study collective synchronization [10]. Since then it has become a paradigmatic model to explain temporal organization in a large variety of natural systems far from thermodynamic equilibrium $[5,11]$. Under some approximations, the KM can be rigorously obtained from Eq. (1). Indeed, when the mutual coupling $K$ of the oscillators is weak, a perturbation treatment permits one to reduce Eq. (1) to a set of $N$ equations for the phases only [1],

$$
\begin{aligned}
\dot{\theta}_{j}= & \omega_{j}+K\left(q_{j}-c\right)+K R\left[\left(1+q_{j} c\right) \sin \left(\Psi-\theta_{j}\right)\right. \\
& \left.-\left(q_{j}-c\right) \cos \left(\Psi-\theta_{j}\right)\right],
\end{aligned}
$$

where $R e^{-i \Psi}=N^{-1} \sum_{k=1}^{N} e^{i \theta_{k}}$ is the complex order parameter. Originally, Kuramoto considered Eq. (1) without reactive coupling and without shear [10]. The resulting phase equation (2) with $c=q_{j}=0$ is the well-known KM. Assuming constant shear, $q_{j}=\hat{q}$, model (2) is equivalent to the so-called Sakaguchi-Kuramoto model $[12,13]$. This can be seen using the definition $\tan \beta_{j}=\left(q_{j}-c\right) /\left(1+q_{j} c\right)$, with $\left|\beta_{j}\right| \leqslant \frac{\pi}{2}$, which permits one to write Eq. (2) in the more compact form,

$$
\dot{\theta_{j}}=\omega_{j}+\frac{\left(1+q_{j} c\right) K}{\cos \beta_{j}}\left[R \sin \left(\Psi-\theta_{j}-\beta_{j}\right)+\sin \beta_{j}\right] .
$$

As in the KM, the Sakaguchi-Kuramoto model shows a transition from incoherence to collective synchronization at large enough values of $K(1+\hat{q} c)$. The synchronized solution can be obtained explicitly if $g(\omega)$ is a Lorentzian distribution.

We recently reported in Ref. [14] that, if shear is distributed according to a certain probability function $h(q)$, the onset of synchronization is prevented when the width of $h(q)$ exceeds a precise threshold. These results were obtained assuming purely dissipative coupling $(c=0)$, and are fully analytic if $g(\omega)$ and $h(q)$ are both Lorentzian. More recently [15], we allowed $\omega$ and $q$ to be nonindependent, but still considering $c=0$.

Our first aim in this paper is to analyze the phase reduction (2) with a general diffusive coupling $(c \neq 0)$ and independent random variables $\omega_{j}$ and $q_{j}$. We will show that, in this case, if $g(\omega)$ and $h(q)$ are both Lorentzian, the onset of synchronization is also prevented beyond a critical value of the width of $h(q)$. In the second part, we address the case of purely reactive coupling, since it has physical relevance in the context of arrays of coupled nanomechanical oscillators [16-18], and in ion chains interacting via Coulomb forces [19]. We will demonstrate that, in this case, the synchronization's critical coupling becomes fully independent of the particular shape of the shear distribution $h(q)$. Finally, we briefly discuss the implications of this result for the KM with random coupling strengths recently studied by Hong and Strogatz [20,21].

\section{GENERAL DIFFUSIVE COUPLING $(c \neq 0)$}

To analyze Eq. (2), we adopt the thermodynamic limit $N \rightarrow$ $\infty$. This allows us to define a probability density function (PDF) for the phases $f(\theta, \omega, q, t)$, such that the complex order 
parameter $r=R e^{-i \Psi}$ is

$$
r(t)=\iint_{-\infty}^{\infty} \int_{0}^{2 \pi} e^{i \theta} f(\theta, \omega, q, t) d \theta d \omega d q .
$$

The evolution of Eq. (2) obeys the continuity equation,

$\partial_{t} f=-\partial_{\theta}\left(\left\{\omega+K(q-c)+\frac{K}{2 i}\left[r e^{-i \theta} C(1-i q)-\right.\right.\right.$ c.c. $\left.\left.]\right\} f\right)$.

Here $C \equiv 1+i c$, and c.c. stands for complex conjugate of the preceding term. Next we expand $f$ in Fourier series as $f(\theta, \omega, q, t)=\frac{1}{2 \pi} g(\omega) h(q) \sum_{l=-\infty}^{\infty} f_{l}(\omega, q, t) e^{i l \theta}$, with $f_{l}=$ $f_{-l}^{*}$ and $f_{0}=1$. Substituting the Fourier series into the continuity equation (3), we obtain the infinite set of integrodifferential equations,

$$
\begin{aligned}
\partial_{t} f_{l}= & -i l[\omega+K(q-c)] f_{l} \\
& +\frac{K l}{2}\left[r^{*} C^{*}(1+i q) f_{l-1}-r C(1-i q) f_{l+1}\right] .
\end{aligned}
$$

The next step is to assume that the asymptotic solutions of the model belong to the family of functions,

$$
f_{l}(\omega, q, t)=\alpha(\omega, q, t)^{l},
$$

a type of ansatz discovered by Ott and Antonsen [22-24]. This solution of Eq. (4) requires $\alpha$ to evolve according to

$$
\begin{aligned}
\partial_{t} \alpha= & -i[\omega+K(q-c)] \alpha \\
& +\frac{K}{2}\left[r^{*} C^{*}(1+i q)-r C(1-i q) \alpha^{2}\right],
\end{aligned}
$$

with

$$
r^{*}(t)=\iint_{-\infty}^{\infty} g(\omega) h(q) \alpha(\omega, q, t) d \omega d q .
$$

A considerable simplification is achieved if $g(\omega)$ and $h(q)$ are chosen to be Lorentzian PDFs,

$$
g(\omega)=\frac{\delta / \pi}{\left(\omega-\omega_{0}\right)^{2}+\delta^{2}}, \quad h(q)=\frac{\gamma / \pi}{\left(q-q_{0}\right)^{2}+\gamma^{2}} .
$$

In this case, the integral (7) can be solved closing the integrals at infinity and using the residue's theorem; notice that $g(\omega)=$ $(2 \pi i)^{-1}\left[\left(\omega-\omega_{0}-i \delta\right)^{-1}-\left(\omega-\omega_{0}+i \delta\right)^{-1}\right]$, likewise for $h(q)$. The important requirement is that the complex function $\alpha$ can be analytically continued from real $\omega$ and $q$ into the complex planes $\omega=\omega_{r}+i \omega_{i}$ and $q=q_{r}+i q_{i}$, inside the integration contours.

It can be shown that $\alpha$ has no singularities in the lower half $\omega$ plane [22]. Regarding the variable $q$, we follow the reasoning in Ref. [14] and find that $\alpha$ is analytic in the lower half complex $q$ plane for $K>0$, and in the upper one for $K<0$. However, now this holds true only if the order parameter satisfies [25]

$$
R<R_{\times}=\frac{1}{\sqrt{1+c^{2}}} .
$$

We assume that states fulfilling this condition are correctly analyzed within this framework. As we show below, the numerical simulations fully confirm the validity of this assumption.
Therefore, using the residue's theorem, the integrals in Eq. (7) give

$$
r^{*}(t)=\alpha\left(\omega=\omega^{p}, q=q^{p}, t\right) \equiv a(t),
$$

where $\omega^{p}=\omega_{0}-i \delta$ and $q^{p}=q_{0} \mp i \gamma$ (for positive and negative $K$, respectively) correspond to the simple poles of the Lorentzian PDFs (8). The infinite set of ordinary differential equations (6) then simply reduces to the single ordinary differential equation with complex variable,

$$
\dot{a}=-i \omega^{p} a+\frac{K}{2} C\left(1-i q^{p}\right)\left(1-|a|^{2}\right) a .
$$

As $a=R e^{-i \Psi}$, the equations for the order parameter inside the manifold defined by Eq. (5) read

$$
\begin{aligned}
& \dot{R}=\left[-\delta+\frac{K}{2}\left(1+c q_{0} \mp \gamma\right)\left(1-R^{2}\right)\right] R, \\
& \dot{\Psi}=\omega_{0}+\frac{K}{2}\left[q_{0}-c(1 \mp \gamma)\right]\left(1-R^{2}\right),
\end{aligned}
$$

which we conjecture are the correct equations for the evolution of the order parameter, as far as condition (9) is fulfilled. From Eq. (11), we find that a synchronized solution bifurcates from incoherence at the critical coupling,

$$
K_{c}=\left\{\begin{array}{lll}
\frac{2 \delta}{1+q_{0} c-\gamma} & \text { if } & 1+q_{0} c>0 \\
\frac{2 \delta}{1+q_{0} c+\gamma} & \text { if } & 1+q_{0} c<0
\end{array}\right.
$$

which only exists if

$$
\gamma<\gamma_{d}=\left|1+q_{0} c\right|
$$

Otherwise, if $\gamma \geqslant \gamma_{d}$, incoherence becomes the only stable state for all $K$; see Fig. 1. This result extends the one found in Ref. [14] for $c=0$ to ensembles of oscillators globally coupled via both dissipative and reactive coupling. However, as is depicted in Fig. 1(a), now the region of stable synchronization is located at positive values of $K$ only if

$$
1+q_{0} c>0
$$

(a)

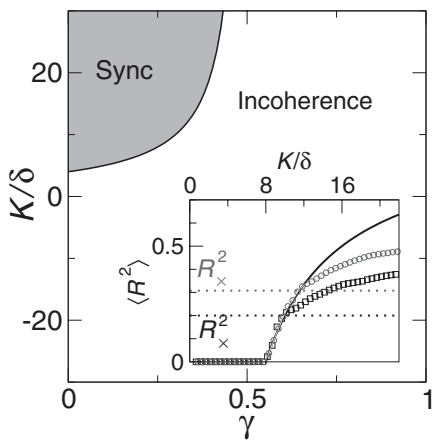

(b)

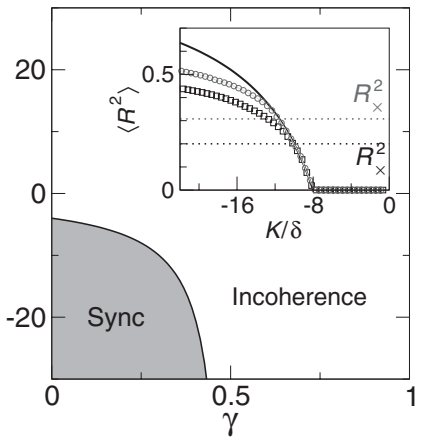

FIG. 1. Phase diagrams with boundary (13) for (a) $q_{0} c=-\frac{1}{2}$ and (b) $q_{0} c=-\frac{3}{2}$. Insets show the numerical results of the timeaveraged quantity $R^{2}$ vs $K / \delta$, with parameters $\gamma=\frac{1}{4}\left(\left|K_{c}\right|=8 \delta\right)$, $\delta=0.1$, and $\omega_{0}=3 .\left\{\omega_{j}, q_{j}\right\}_{j=1, \ldots, N}$ were deterministically selected to represent the distribution (8) with $N=14400$ oscillators. The data sets correspond to two different combinations of $q_{0}$ and $c$, with $c=2$ $\left(R_{\times}^{2}=\frac{1}{5}\right)$ and $c=\frac{3}{2}\left(R_{\times}^{2}=\frac{4}{13}\right)$. The solid line is Eq. (16). 
This inequality is the well-known Benjamin-Feir-Newell criterion for the stability of plane waves in the homogeneous CGLE [1,3,13,26,27], which is valid in any dimension (infinite in the present case). Finally, we also find that the order parameter of the partially synchronized solution follows:

$$
R^{2}=\frac{K-K_{c}}{K}, \quad \text { with } \quad R<R_{\times},
$$

exactly as in the KM with Lorentzian $g(\omega)$ [1]. However, now this formula holds only up to $R_{\times}$; recall Eq. (9). The insets in Fig. 1 show numerical simulations that confirm the validity of Eq. (16). Remarkably, our numerical simulations indicate that $R$ departs from Eq. (16) precisely when $R$ exceeds $R_{\times}$.

Unfortunately, there is no straightforward theoretical extension of these results to more general distributions $g(\omega)$ and $h(q)$, but still some reasonable conjectures can be raised as follows.

(i) The flip of the synchronization region when $1+q_{0} c$ changes sign — compare Figs. 1(a) and 1(b)—is a consequence of the Benjamin-Feir-Newell criterion (15) and it can be supposed to be a general feature of Eq. (2).

(ii) Although it seems difficult to prove, the divergence of $K_{c}$ at a critical value of the shear diversity Eq. (14) is likely to be a general property, as it happens in the purely dissipative case $(c=0)$ [14].

(iii) For certain distributions and parameter values, and as a consequence of the persistence of a fully synchronized solution $\theta_{j}=\Psi$ located at $|K| g\left(\omega_{0}\right) \rightarrow \infty$, stable synchronization and incoherence should coexist at large $K$ values (as it occurs for $c=0$ and Gaussian distributions [14]). Note that infinitesimal perturbations obey $\dot{\delta} \dot{\theta}_{j}=\frac{K}{N}\left(1+q_{j} c\right)$ $\left[(1-N) \delta \theta_{j}+\sum_{l \neq j} \delta \theta_{l}\right]$. The Jacobian matrix has always one trivial zero eigenvalue. If $c=0$ and $K$ positive, the remaining eigenvalues are negative, and the fixed point is stable irrespective of the width of $h(q)$. However, if $c \neq 0$, the fixed point becomes a saddle when the $q_{j}$ 's exceed some degree of heterogeneity, and hence its continuation at finite $K$ is not an attractor either. In sum, under a large enough heterogeneity of shear, incoherence should be the only attractor at all $K$ values; however, if $c=0$, synchronization coexists with incoherence at large enough $K$-provided $h(q)$ is not heavy-tailed; see Ref. [14].

\section{PURELY REACTIVE COUPLING}

For the remainder of this paper, we will concentrate on the case of purely reactive coupling. Motivated by the dynamics of nanoscale mechanical oscillator arrays, this problem was analyzed in detail by Cross et al. $[16,17]$ with a coupling of the form $i \frac{\kappa}{N} \sum_{k}\left(z_{k}-z_{j}\right)$ in Eq. (1), and without shear diversity. To investigate the effect of shear diversity, we first write the phase model (2) without dissipative coupling. Substituting $c=\kappa / K$ in Eq. (2) and letting $K \rightarrow 0$ yields

$$
\dot{\theta}_{j}=\omega_{j}-\kappa+\kappa R\left[q_{j} \sin \left(\Psi-\theta_{j}\right)+\cos \left(\Psi-\theta_{j}\right)\right],
$$

where $\kappa$ is now the total reactive coupling. Then, Eq. (13) suggests that in this limit the critical coupling is

$$
\kappa_{c}=\frac{2 \delta}{q_{0}},
$$

that remarkably depends on $q_{0}$ but is independent of the amount of heterogeneity $\gamma$.

The derivation of Eq. (18) is not rigorous because $R_{\times}=0$ in this limit, and condition (9) is not fulfilled. Therefore, to confirm the validity of Eq. (18) and to determine how this result generalizes to other distributions, next we perform the linear stability analysis of the incoherent state of Eq. (17) [28]. In the incoherent state all modes $f_{l}$, save the trivial one $f_{0}=1$, vanish. The equation for the Fourier modes, related to Eq. (4), is

$$
\partial_{t} f_{l}=-i l(\omega-\kappa) f_{l}+\frac{\kappa l}{2}\left[r^{*}(q-i) f_{l-1}-r(q+i) f_{l+1}\right] .
$$

Linearizing this equation about the incoherent state, we find that the only potentially unstable mode is the $l=1$,

$$
\begin{aligned}
\partial_{t} f_{1}= & -i(\omega-\kappa) f_{1} \\
& +\frac{\kappa}{2}(q-i) \iint_{-\infty}^{\infty} f_{1}\left(\omega^{\prime}, q^{\prime}, t\right) g\left(\omega^{\prime}\right) h\left(q^{\prime}\right) d \omega^{\prime} d q^{\prime} .
\end{aligned}
$$

Let $f_{1}(\omega, q, t)=b(\omega, q) e^{\lambda t}$, and neglect the trivial solution $b=0$. Invoking self-consistency and separating $\lambda$ into its real and imaginary parts $\left(\lambda=\lambda_{r}+i \lambda_{i}\right)$ yields

$$
\frac{2}{\kappa}=\iint_{-\infty}^{\infty} \frac{(q-i)\left[\lambda_{r}-i\left(\omega-\kappa+\lambda_{i}\right)\right]}{\lambda_{r}^{2}+\left(\omega-\kappa+\lambda_{i}\right)^{2}} g(\omega) h(q) d \omega d q .
$$

The interesting feature in the right-hand side of this equation is that the integration over $q$ is trivial and the result does not depend on the particular shape of $h(q)$. Performing the limit $\lambda_{r} \rightarrow 0^{+}$to obtain the stability threshold $\kappa_{c}$ yields

$$
\frac{2}{\kappa_{c}}=\left(q_{0}-i\right)\left[\pi g\left(\kappa_{c}-\lambda_{i}\right)-i \int_{-\infty}^{\infty} \frac{g(\omega)}{\omega-\kappa_{c}+\lambda_{i}} d \omega\right],
$$

that only depends on $h(q)$ through its mean value $q_{0}$ (defined as principal value if required). Finally, splitting Eq. (19) into its real and imaginary parts, we obtain a system of two equations for the unknowns $\kappa_{c}$ and $\lambda_{i}$

$$
\begin{aligned}
\left(1+q_{0}^{2}\right) \pi g\left(\kappa_{c}-\lambda_{i}\right) & =\frac{2 q_{0}}{\kappa_{c}}, \\
\left(1+q_{0}^{2}\right) \int_{-\infty}^{\infty} \frac{g(\omega)}{\omega-\kappa_{c}+\lambda_{i}} d \omega & =-\frac{2}{\kappa_{c}} .
\end{aligned}
$$

These equations can be solved for Lorentzian $g(\omega)$, and indeed we recover the boundary (18). However, note that now this result is stronger, since we have not imposed any constraint on the shape of $h(q)$. Here $h(q)$ can be any distribution of mean $q_{0}$. Additionally, an explicit value for $\kappa_{c}$ can be easily obtained from Eq. (20) if $g(\omega)$ is a uniform (top-hat) distribution. These results for Lorentzian and uniform $g(\omega)$ are in agreement with those obtained in Ref. [17] with $h(q)=\delta\left(q-q_{0}\right)$. This confirms that the phase equation is indeed a good approximation of the amplitude equation in the limit of weak coupling and narrow frequency distributions.

Figure 2(a) displays a phase diagram with the boundary (18), and Fig. 2(b) shows the time average $\left\langle R^{2}\right\rangle$ obtained from numerical simulations using different distributions $h(q)$ with common $q_{0}$ values. As expected, in Fig. 2(b), the transition between incoherence and synchronization occurs at the same value of $\kappa / \delta$, irrespective of the distribution type. 
(a)

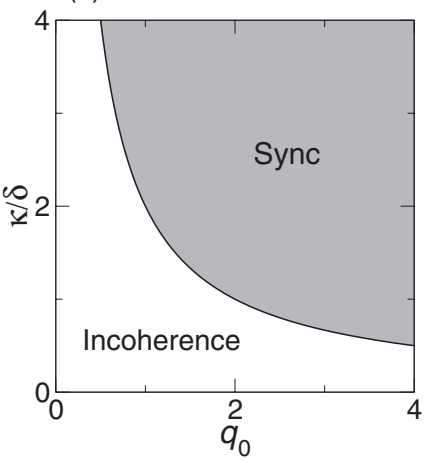

FIG. 2. (a) Phase diagram with boundary (18) in the case of purely reactive coupling and $g(\omega)$ Lorentzian. (b) Time averages $\left\langle R^{2}\right\rangle$ obtained from numerical simulations with $q_{0}=2, \delta=1$, and $N=40000$, and for different distributions of $q$ : Gaussian with variance $v^{2}=1$; symmetric bidelta: $h(q)=\frac{1}{2} \delta(q-3)+\frac{1}{2} \delta(q-1)$; asymmetric bidelta: $h(q)=\frac{3}{4} \delta(q-3)+\frac{1}{4} \delta(q+1)$; Lorentzian with $\gamma=1$; and bimodal: $h(q)=\frac{1 /(2 \pi)}{\left[q-\left(q_{0}-5 / 2\right)\right]^{2}+1}+\frac{1 /(2 \pi)}{\left[q-\left(q_{0}+5 / 2\right)\right]^{2}+1}$. In all cases, the critical coupling is at $\kappa_{c} / \delta=1$, as predicted by Eq. (18).

Finally, we point out an interesting similarity between Eq. (17) and the model recently studied by Hong and Strogatz $[20,21]$, which in our notation reads $\dot{\theta}_{j}=\omega_{j}+q_{j} R \sin (\Psi-$ $\theta_{j}$ ). Note that here $q_{j}$ acts as a distributed coupling strength. Performing a stability analysis like we did above, we obtain

that the stability border of incoherence satisfies $2=\pi q_{0} g\left(\omega_{0}\right)$, if $g(\omega)$ is unimodal and symmetric. Again, we obtain a formula that depends on the mean of $h(q)$, but not on its shape. This result reproduces the classical Kuramoto relation for uniform all-to-all coupling $\left[h(q)=\delta\left(q-q_{0}\right)\right]$, and the critical point found in Eq. (12) of Ref. [20] for Lorentzian $g(\omega)$ and bidelta $h(q)$.

\section{CONCLUSIONS}

We have reported on exact results that extend the phase models of Kuramoto and Sakaguchi $[10,12]$ to situations where shear is distributed. In contrast to the recent work [14], here the coupling is not purely dissipative but also contains a reactive component $c$. In this case, we also find that shear diversity prevents the onset of collective synchronization, but the Benjamin-Feir-Newell criterion determines now if the region of synchronization is located at positive or negative values of $K$. Finally, we have obtained a remarkable result when the coupling is purely reactive: the stability threshold of incoherence depends on the mean shear $q_{0}$, while the shear diversity becomes irrelevant.

\section{ACKNOWLEDGMENTS}

Financial support from the Ministerio de Ciencia e Innovación (Spain) under Projects No. FIS2009-12964-C05-05 and No. SAF2010-16085 is acknowledged.
[1] Y. Kuramoto, Chemical Oscillations, Waves, and Turbulence (Springer-Verlag, Berlin, 1984).

[2] J. D. Murray, Mathematical Biology, Interdisciplinary Applied Mathematics (Springer, New York, 2003).

[3] M. C. Cross and P. C. Hohenberg, Rev. Mod. Phys. 65, 851 (1993).

[4] A. T. Winfree, The Geometry of Biological Time (Springer, New York, 1980).

[5] A. S. Pikovsky, M. G. Rosenblum, and J. Kurths, Synchronization, a Universal Concept in Nonlinear Sciences (Cambridge University Press, Cambridge, UK, 2001).

[6] S. H. Strogatz, Sync: The Emerging Science of Spontaneous Order (Hyperion Press, New York, 2003).

[7] P. C. Matthews, R. E. Mirollo, and S. H. Strogatz, Physica D 52, 293 (1991).

[8] H. Daido and K. Nakanishi, Phys. Rev. Lett. 96, 054101 (2006).

[9] See Refs. [1,5,29] for the case of two coupled Stuart-Landau oscillators, and Refs. $[13,16,17,26]$ for the mean-field CGLE.

[10] Y. Kuramoto, in International Symposium on Mathematical Problems in Theoretical Physics, Lecture Notes in Physics, Vol. 39, edited by H. Araki (Springer, Berlin, 1975), pp. $420-422$.

[11] J. A. Acebrón et al., Rev. Mod. Phys. 77, 137 (2005).

[12] H. Sakaguchi and Y. Kuramoto, Prog. Theor. Phys. 76, 576 (1986).

[13] N. Nakagawa and Y. Kuramoto, Prog. Theor. Phys. 89, 313 (1993).

[14] E. Montbrió and D. Pazó, Phys. Rev. Lett. 106, 254101 (2011).
[15] D. Pazó and E. Montbrió, Europhys. Lett. 95, 60007 (2011).

[16] M. C. Cross, A. Zumdieck, R. Lifshitz, and J. L. Rogers, Phys. Rev. Lett. 93, 224101 (2004).

[17] M. C. Cross, J. L. Rogers, R. Lifshitz, and A. Zumdieck, Phys. Rev. E 73, 036205 (2006).

[18] S.-B. Shim, M. Imboden, and P. Mohanty, Science 316, 95 (2007).

[19] T. E. Lee and M. C. Cross, Phys. Rev. Lett. 106, 143001 (2011).

[20] H. Hong and S. H. Strogatz, Phys. Rev. Lett. 106, 054102 (2011).

[21] H. Hong and S. H. Strogatz, Phys. Rev. E 84, 046202 (2011).

[22] E. Ott and T. M. Antonsen, Chaos 18, 037113 (2008).

[23] E. Ott and T. M. Antonsen, Chaos 19, 023117 (2009).

[24] E. Ott, B. R. Hunt, and T. M. Antonsen, Chaos 21, 025112 (2011)

[25] In the semicircular path at infinity $(|q| \rightarrow \infty)$ parametrized by $\vartheta, q=|q| e^{i \vartheta}$, the evolution equation of $|\alpha|$ evaluated at $|\alpha|=$ 1 yields at leading order $\partial_{t}|\alpha|=K(1-R|C| \cos \chi)|q| \sin \vartheta$, where $|\alpha|=\alpha e^{-i \psi}, C=|C| e^{i \zeta}$, and $\chi=\psi(q, t)-\Psi(t)-\zeta$. Thus the condition $\partial_{t}|\alpha|<0$ assuring that Eq. (5) remains finite is fulfilled if $R<R_{\times}=|C|^{-1}$, in $\vartheta \in(0, \pi)$ for $K<0$, and in $\vartheta \in(-\pi, 0)$ for $K>0$.

[26] V. Hakim and W. J. Rappel, Phys. Rev. A 46, R7347 (1992).

[27] I. S. Aranson and L. Kramer, Rev. Mod. Phys. 74, 99 (2002).

[28] S. H. Strogatz and R. E. Mirollo, J. Stat. Phys. 63, 613 (1991).

[29] D. G. Aronson, G. B. Ermentrout, and N. Kopell, Physica D 41, 403 (1990). 